Proceedings of the 2013 Winter Simulation Conference

R. Pasupathy, S.-H. Kim, A. Tolk, R. Hill, and M. E. Kuhl, eds

\title{
CONTINUOUS VARIABLE CONTROL APPROACH FOR HOME CARE CREW SCHEDULING
}

\author{
Seokgi Lee \\ Yuncheol Kang \\ Vittaldas V. Prabhu \\ Harold and Inge Marcus Department of Industrial and Manufacturing Engineering \\ The Pennsylvania State University \\ University Park, PA 16802 USA
}

\begin{abstract}
The home care crew scheduling problem (HCCSP) is defined as a dynamic routing and scheduling problem with caretakers' fixed appointments, and therefore has many similarities with the vehicle routing problem with time windows. Considering frequent demand changes regarding resource priorities, appointment alterations, and time windows in HCCSP, the control theoretic approach with discrete event distributed simulation provide substantial benefits by offering real-time response to demand changes. We develop dynamic models for HCCSP with dynamic patient appointments, and explain dynamics that span from controlling crew work times to home-visit scheduling. Also, the real-time feedback control algorithm is proposed to solve HCCSP, where it is performed based on the time-scaled approach that possibly eliminates the need for directly synchronizing events and thereby eliminates the complexity associated with discrete event distributed simulation approaches.
\end{abstract}

\section{INTRODUCTION}

The demand for long-term care in the United States was 15 million in 2000 and is expected to nearly double to 27 million in 2050 (Bercovitz et al. 2011). The majority of this demand takes the form of care at home ('home care') rather than hospitalization. By definition, home care is supportive care allowing individuals to remain in their homes or communities, and ranges from professional home health service, such as treatment and injections, dressing changes, and supervision of medication, to other routine household chores, such as eating, toileting, housekeeping, laundry and mending, respite care, and transportation (Smith et al. 2000). Generally, such home care services are provided by home care caregivers, including home care nurses, professional therapists, and unskilled helpers who support a resident's activities of daily living (ADL).

Given the significant increase in demand, the home care industry is faced with shortages of home care caregivers. In 2007, only 160,700 home care caregivers were employed by home care agencies, and recruitment of additional caregivers or retention of current workers has become a critical issue, due to their poor working conditions (Bercovitz et al. 2011). Moreover, home care agencies may cover 5,000 square miles as a service region, and caregivers drive 5 billion miles each year to supply home health care services (Bennett 2010). Such challenges now require the home care industry to develop very cost-efficient planning and scheduling.

Theoretically, scheduling daily home care visits has been recognized as an important operations research (OR) problem in the home care area and it has been solved using various OR approaches as the home care crew scheduling problem (HCCSP). Basically, HCCSP is to determine the best routing sched- 


\section{Lee, Kang, and Prabhu}

ules for caregivers, with the objective of reducing total travel cost and maximizing service level (Rasmussen et al. 2012). Overall problem definitions and approaches are similar to the capacitated vehicle routing problem (CVRP), except for several temporal or spatial constraints related to tasks, caregivers, and patients. Therefore, various optimization and meta-heuristic approaches, along with the use of advanced information technology, have been developed to solve HCCSP.

Dynamic home care crew routing and scheduling problems with fixed appointment times are solved by a new rolling horizon capacity-based heuristic approach (Bennett and Erera 2011). Borsani et al. (2006) consider home care crew assignment and scheduling problems under the Italian home care environment using a linear integer programming approach, mainly focusing on the scheduling problem rather than the assignment problem. Rasmussen et al. (2012) modeled the HCCSP as a set partitioning problem and solved it using a branch-and-price solution algorithm, considering the way to minimize overall travel costs while maximizing the home care service level by multi-criteria objective function. In case of metaheuristic approaches, Bertels and Fahle (2006) applied linear programming, constraint programming, and a variety of meta-heuristic algorithms to solve the home health care staff rostering and routing problems. The Particle Swarm Optimization (PSO) technique, which is based on a meta-heuristic approach, was developed to solve HCCSP (Akjiratikarl et al. 2007). As another heuristic approach, Misir et al. (2010) used a hyper-heuristics approach to sort out proper heuristic sets for solving. Along with those optimization and heuristic approaches, information technology plays a key role in efficiently managing home care resources. For example, a systematic approach was used to develop an Information and Communication Technology (ICT) system for scheduling home care units in Sweden (Eveborn et al. 2009); a spatial decision support system with Geographic Information Systems (GIS) has also been developed (Begur et al. 1997).

In this paper, we develop continuous-time dynamic models for controlling the arrival time of a care request from a resident and a caregiver simultaneously, and a distributed feedback control algorithm based on discrete event simulation for HCCSP. The distributed control approach using the integral control structure of dynamic models results in algorithmic benefits, dramatically decreasing computational complexity and therefore enabling the home care system to respond to the dynamic home care environment. The rest of this paper is organized as follows: In section 2, HCCSP is defined and corresponding dynamic models are developed. In section 3, dynamic behavior of the proposed model is illustrated. The distributed feedback control algorithm is presented and its performance is tested using various examples in section 4 . Lastly, performance evaluation of the proposed feedback control algorithm is explained in section 5.

\section{PROBLEM DEFINITION}

In this section we describe the Home Care Crew Scheduling Problem (HCCSP) in more detail, and explain a few underlying assumptions.

Residents, as service recipients, are the people who need home care services. When they are registered to a home care agency they specify the needed tasks (i.e. the kinds of tasks and any time constraints) and supply their home address, where they will receive the care services. Their home address information is converted into a distance matrix, which contains relative distances between the resident's and caregiver's location. Residents may also have preferred caregivers. Generally, residents often prefer for the same caregiver to come to them each day (Rasmussen et al. 2012). Thus, it is desirable to match the more favorable caregiver to the resident when there are two or more available caregivers. This constraint is considered as part of the algorithm described here.

The HCCSP can be expressed using a complete graph $G=(\mathcal{N}, \mathcal{A})$ where $\mathcal{N}=\{0,1,2, \ldots, \mathrm{n}\}$ is the vertex set and $\mathcal{A}$ is the arc set among vertices. Vertex set $\mathcal{N}_{0}=\mathcal{N} \backslash\{0\}$ corresponds to locations of residents or residents themselves, and vertex 0 indicates the home care agency, where caregivers may begin their schedule. Each resident can request a one or more tasks through one or more caregivers. The travel time between the resident $i-1$ and $i$ is denoted as $r_{i-1, i},(i-1, i) \in \mathcal{A}$. (Here, $r_{0,1}$ indicates the travel time between the home care agency and the first resident.) From a timing perspective, each resident 


\section{Lee, Kang, and Prabhu}

$i \in \mathcal{N}_{0}$ is associated with a nonnegative service time $\varphi_{i}$ to be delivered within a resident-requested time window $\left[x_{i}, y_{i}\right]$ which is a specified time period in which the caregiver must arrive and start services.

Caregivers are the people who provide care services. Caregivers include home care nurses, professional assistants, or unskilled helpers. Each caregiver may have a different competence level. For example, homecare nurses may assist in clinically related activities such as dialysis, whereas unskilled helpers cannot. Also, each caregiver may have specific available working hours. In particular, some homecare workers may not have the same primary duties of homecare nurses. Such a caregiver set can be defined as $\mathcal{M}=\{1,2, \ldots, m\}$, in which each caregiver $j \in \mathcal{M}$ has a different working time limit, $h_{j}$, per a planning horizon, and is initially available at the home care agency.

In HCCSP, the task set $\mathcal{K}=\{1,2, \ldots, k\}$ consists of care services that need to be carried out by caregivers. Such tasks may vary in terms of required competence level. Some tasks may include low skill level services, such as changing bed linens or doing laundry, while the other tasks may require a high skill level, such as dialysis or professional therapy. Also, a certain task may require two or more caregivers, such as lifting a resident from a bed into a wheel chair. In this case, at least two caregivers need to come to the resident's home at the same time to fulfill the task. Moreover, a certain task may have additional time constraints in terms of starting time. From the lifting example above, such a task is normally required right after a caregiver wakes up the resident in the morning. Consequently, a task may have many aspects when we consider various scenarios; the HCCSP may have to include such complex constraints to produce more realistic schedules.

The objective of the HCCSP is to find caregiver routes that minimize transportation costs associated with fulfillment of requested tasks. This time-related metric is represented by the sum of earliness and tardiness for the resident-demanded time window.

Considering all $n$ residents and their time windows $\left[x_{i}, y_{i}\right], i \in \mathcal{N}_{0}$, the sum of earliness and tardiness is calculated by

$$
\sum_{i \in \mathcal{N}_{0}}\left(E_{i}+T_{i}\right)
$$

where $E_{i}$ and $T_{i}$ are the earliness and tardiness of the $i$ th resident such that

$$
\begin{aligned}
& E_{i}=\max \left[0, x_{i}-c_{i}\right]=\left[x_{i}-c_{i}\right]^{+}, \\
& T_{i}=\max \left[0, c_{i}-y_{i}\right]=\left[c_{i}-y_{i}\right]^{+},
\end{aligned}
$$

where $c_{i}$ is the arrival time of a caregiver at the $i$ th resident's location.

Finally, the HCCSP uses mean-squared due-date deviation (MSD) of the earliness and tardiness such that

$$
\sqrt{\frac{\sum_{i \in \mathcal{N}_{0}}\left(E_{i}+T_{i}\right)^{2}}{n}}
$$

which measures the average difference between the resident-requested start time of service and the actual start time. 


\section{DYNAMIC MODEL}

One objective of HCCSP is to determine a caregiver's departure time to minimize the discrepancy between the requested service starting time and the estimated service starting time on the given task. In this paper, we suggest a departure time controller to deal with this objective function. In particular, the departure time controller is modeled as a distributed feedback control model, which can dynamically adjust the departure times of caregivers with respect to each task, to minimize due date deviation. Finally, the overall chronological sequence of the departure times on all tasks of residents decides a crew schedule.

To develop a departure time controller of the HCCSP, let us define the task set $S_{i} \subseteq \mathcal{K}$, which is the ordered subset of $\mathcal{K}$, indicating the list of multiple tasks requested by the resident $i$, and $S_{i, k} \in S_{i}$ is the $k$ th task of the resident $i$. Then we can define the following time-variant variables used in the dynamic model: for the $k$ th task of the resident $i, a_{S_{i, k}}(t)$ is the departure time of a care giver, $c_{S_{i, k}}(t)$ is the arrival time of a caregiver at resident $i, \varphi_{S_{i, k}}(t)$ is the service time, and $q_{S_{i, k}}(t)$ is the queuing time. The resident-requested time window is simply defined as $\left[x_{i}, y_{i}\right]$ in section 2 , but now it is redefined as $\left[x_{S_{i, k}}\right.$, $\left.y_{S_{i, k}}\right]$, breaking down into a task of each resident. Lastly, the travel time between the $(i-1)$ th resident and the $i$ th resident is denoted by $p_{i-1, i}(t)$.

We adjust $a_{S_{i, k}}(t)$ by the departure time controller in proportion to the size of earliness or tardiness in Equation (2) and (3). Thus, the departure time dynamic is explained by

$$
\dot{a}_{S_{i, k}}(t)=\psi_{S_{i, k}}\left(\left[x_{S_{i, k}}-c_{S_{i, k}}(t)\right]^{+}-\left[c_{S_{i, k}}(t)-y_{S_{i, k}}\right]^{+}\right)
$$

where $\psi_{S_{i, k}}$ is the control gain.

Here, the departure time controller (5) gives equal penalties for both earliness and tardiness to minimize the due-date deviation for each task. In case of a feasible due date, the due-date deviation, $\left[x_{S_{i, k}}-\right.$ $\left.c_{S_{i, k}}(t)\right]^{+}-\left[c_{S_{i, k}}(t)-y_{S_{i, k}}\right]^{+}$, asymptotically becomes zero, otherwise the controller makes the timeaverage of the due-date deviation zero. Also, in the case of multiple tasks requested by a single resident, the departure times of corresponding tasks are chronologically ordered and therefore the following relationship must to be satisfied:

$$
a_{S_{i, 1}}(t)<a_{S_{i, 2}}(t)<\cdots<a_{S_{i,\left|S_{i}\right|}}(t)
$$

The arrival time of a caregiver, $c_{S_{i, k}}(t)$, is calculated by

$$
c_{S_{i, k}}(t)=a_{S_{i, k}}(t)+q_{S_{i, k}}(t)+p_{i-1, i}(t)+\sum_{w=1}^{\left|S_{i}\right|} \varphi_{S_{i, w}}(t)
$$

where the queuing time, $q_{S_{i, k}}(t)$ is given by

$$
q_{S_{i, k}}(t)=a_{S_{1, k}}(t)+\sum_{u=1}^{i-1}\left(p_{u-1, u}+\sum_{w=1}^{\left|S_{u}\right|} \varphi_{S_{u, w}}(t)\right)-a_{S_{i, k}}(t),
$$




$$
a_{S_{i, k}}(t)<a_{S_{1, k}}(t)+\sum_{u=1}^{i-1}\left(p_{u-1, u}+\sum_{w=1}^{\left|S_{u}\right|} \varphi_{S_{u, w}}(t)\right)
$$

otherwise zero.

Substituting Equation (8) into (7) yields

$$
c_{S_{i, k}}(t)=a_{S_{1, k}}(t)+\sum_{u=1}^{i}\left(p_{u-1, u}+\sum_{w=1}^{\left|S_{u}\right|} \varphi_{S_{u, w}}(t)\right) .
$$

It should be noted that changing the sequence of departure times results in variations of the crew visiting sequence based on first-come-first-serve (FIFO) discipline, and therefore the completion time in Equation (9) becomes a highly nonlinear function of the departure time with discontinuities, even though it appears to be affected only by the first component, $a_{S_{1, k}}(t)$.

Furthermore, controlling departure times of multiple residents results in a multivariable control system with highly nonlinear coupling between departure and completion times. Using feedback in a local departure time controller for each component, these nonlinearities can be ignored and aggregated, provided adequate iterations of the controller can be executed. Hence, we use the following integral control law for departure time control:

$$
a_{S_{i, k}}(t)=\psi_{S_{i, k}} \int_{0}^{t}\left\{\left[x_{S_{i, k}}-c_{S_{i, k}}(t)\right]^{+}-\left[c_{S_{i, k}}(t)-y_{S_{i, k}}\right]^{+}\right\} d \tau+a_{S_{i, k}}(0)
$$

where $c_{S_{i, k}}(t)$ is calculated by Equation (10) and $a_{S_{i, k}}(0)$ is an arbitrary initial departure time for resident $i$.

\section{DISTRIBUTED FEEDBACK CONTROL ALGORITHM}

As we explained in the dynamic model, a chronological sequence of the departure times for each task decides a final home care crew routing schedule; it can be obtained by the feedback control algorithm, which is performed based on the time-scaled discrete event distributed simulation. In such a simulation model, each entity on the homecare crew service environment will have corresponding virtual entities of a caregiver and a task in all respects, and therefore the following advantages (Prabhu and Duffie 1995):

- The simulation model can provide a high level of detail and accuracy because it is a replica of the control system software.

- The distributed simulation model results in distribution of information which increase fault-tolerance by eliminating single point failures.

- Development and modification of the simulation model can be made inexpensively and immediately.

Continuous variables in the departure time controller (11) are discretized for computer implementation as follows:

$$
a_{S_{i, k}}(T)=\psi_{S_{i, k}} \Delta\left(\left[x_{S_{i, k}}-c_{S_{i, k}}(T-1)\right]^{+}+\left[c_{S_{i, k}}(T-1)-y_{S_{i, k}}\right]^{+}\right)+a_{S_{i, k}}(T-1),
$$




\section{Lee, Kang, and Prabhu}

where $\Delta$ is the discrete time step for integration, and the departure time and the arrival time are denoted in the $T$ th time step, respectively.

When multiple caregivers are staffed for resident care, cost-effective task assignment for each caregiver plays a significant role in improving the home care crew routing procedures, because it is essential to deciding crew routing schedules and corresponding travel costs. To define caregiver assignment rules used in the feedback control algorithm, first we define the following concepts: (i) $\mathcal{M}_{i}^{P} \subseteq \mathcal{M}$ is a caregiver set whose elements are the list of caregivers who are personally preferred by the resident $i$. (ii) $\mathcal{M}_{i}^{L} \subseteq \mathcal{M}$ is a caregiver set consisting of caregivers who have the specific skill set needed by the resident $i$. (iii) $\mathcal{M}_{i}^{T} \subseteq \mathcal{M}$ consists of caregivers who can satisfy the time window requested by the resident $\mathrm{i}$. Based on these subset concepts, caregiver assignment rules for resident $i$ are explained as follows:

- Rule 1: Considering spatial and temporal constraints: choose a caregiver who can satisfy the time window of the resident $i$ and arrive at his or her location most rapidly, $\left(\mathcal{M}_{i}^{P}=\emptyset\right.$ and $\left.\mathcal{M}_{i}^{L}=\emptyset\right)$.

- Rule 2: Considering preference constraint: choose a caregiver who is personally preferred by resident $i$, considering spatial and temporal constraints simultaneously, $\left(\mathcal{M}_{i}^{P} \neq \varnothing\right.$ and $\left.\mathcal{M}_{i}^{L}=\varnothing\right)$.

- Rule 3: Considering skill constraint: choose a caregiver who has the specific skill set demanded by the resident $i$, considering spatial and temporal constraints simultaneously, $\left(\mathcal{M}_{i}^{P}=\emptyset\right.$ and $\left.\mathcal{M}_{i}^{L} \neq \varnothing\right)$.

Finally, we develop a distributed feedback control algorithm with simultaneous task assignment and crew scheduling functions, considering the multiple caregivers, as follows:

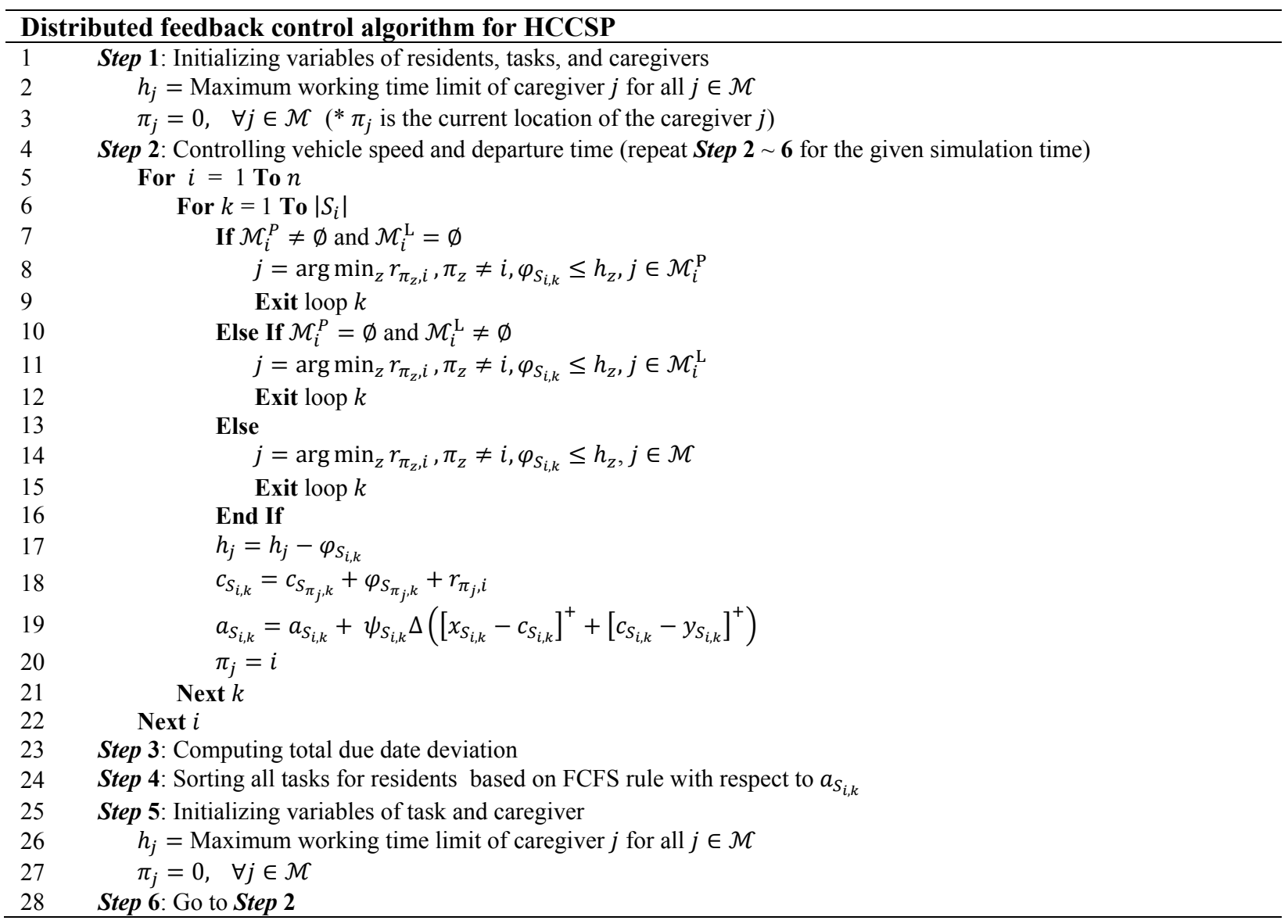




\section{Lee, Kang, and Prabhu}

Table 1: 5-resident data set

\begin{tabular}{|c|c|c|c|c|c|}
\hline Resident id $(\boldsymbol{i})$ & Task id $(\boldsymbol{k})$ & Location id & $\boldsymbol{x}_{\boldsymbol{S}_{\boldsymbol{i} \boldsymbol{k}}}$ & $\boldsymbol{y}_{\boldsymbol{S}_{\boldsymbol{i}, \boldsymbol{k}}}$ & $\boldsymbol{\varphi}_{\boldsymbol{S}_{\boldsymbol{i} \boldsymbol{k}}}$ \\
\hline 1 & 1 & 1 & 4 & 5 & 1 \\
\hline 2 & 1 & 2 & 9 & 10 & 1 \\
\hline 2 & 2 & 2 & 9 & 10 & 1 \\
\hline 3 & 1 & 3 & 10 & 12 & 1 \\
\hline 4 & 1 & 4 & 19 & 20 & 1 \\
\hline
\end{tabular}

The distributed feedback control algorithm has two main functions: (i) task assignment into multiple caregivers (line 6 16) and (iii) departure time control in multiple routes (line 19). The specific algorithm procedure is as follow. First, current locations, $\pi_{j}, \forall j \in \mathcal{M}$, and maximum working times of all caregivers $\left(\pi_{j}, \forall j \in \mathcal{M}\right)$ are initialized (line 2 3). In the control loop (line 5 22), a caregiver is assigned to the given task of a resident, considering (i) resident's preference (line 7 9), (ii) professional skill sets of a caregiver regarding a specific task (line 10 12), or (iii) only the spatial and temporal constraints, and corresponding service time is subtracted from the current working time (capacity) limit of a caregiver (line 17). After anticipating the arrival time at the next resident's location (line 18), the departure time is adjusted by the departure time controller based on the due date deviation (line 19). For every repetition of steps $2 \sim$ 6 , the best crew routing schedule in terms of the given objective functions is saved, and the final result is obtained after the algorithm terminates. In the next section, the distributed feedback control algorithm for HCCSP is evaluated using various problem sets consisting of different conditions of tasks and residents.

\section{PERFORMANCE OF THE DISTRIBUTED FEEDBACK CONTROL ALGORITHM}

In this section, we explain how the home care crew schedule is determined by the proposed distributed feedback control algorithm, using two data sets: (i) preference and skill set constraints do not exist, and (ii) both constraints exist. The test data set consists of 5 residents and each of them needs a single task, except the second resident, who has two different tasks, as shown in Table 1. Particularly, in the second test data set, the fifth resident has a personal caregiver preference for the second caregiver, and the third resident needs special care which can be performed only by the third caregiver. The distributed feedback control algorithm is performed using the following initial set of parameters variables: for all $(i, k)$, the control gain, $\psi_{S_{i, k}}=0.01$, and the total number of iterations (simulation time) is 1000 . Also, the initial departure times of all (i, k) are equally set as $a_{S_{i, k}}(0)=0.0$.

The simulation results of each case are illustrated in Figures 1 and 2, respectively. In each figure, a number in a circle indicates a resident id (zero indicates the agency center), and its location with respect to the vertical line (planning horizon - unit time) indicates its time window position; a number in a brackets is a caregiver id; the horizontal line indicates a caregiver assignment phase decided by resident and caregiver loops in the feedback control algorithm. A gray circle is a selected resident at each assignment phase, and a number beside an arrow line is a travel time between two residents' locations. Furthermore, selected caregivers at each assignment phase and applied assignment rules are shown in the bottom tables, respectively.

If no constraint on caregiver preference and skill sets exists, the algorithm only considers spatial and temporal conditions to assign a caregiver to a task for a resident, as shown in Figure 1. For example, at the first and second caregiver assignment phase, caregiver 1 is assigned into $S_{1,1}$ and $S_{3,1}$, because it is anticipated that these time windows $([4,5]$ and $[10,12])$ are all satisfied by caregiver 1 without earliness or lateness (temporal condition), and therefore caregiver 1, who will be the first to arrive at each resident's location (spatial condition) is selected. In the phase 3 , however, the time window of $S_{2,1}([9,10])$ cannot be promised by the caregiver 1, who is supposed to arrive at the location of resident 3 after the time window of $S_{2,1}$. Thus, another caregiver, caregiver 2 , is assigned into $S_{2,1}$ to satisfy the temporal 
condition. In this way, the crew routing schedule of each caregiver is decided by considering the temporal and spatial conditions simultaneously, and the final results are (center $\rightarrow S_{1,1} \rightarrow S_{3,1} \rightarrow S_{5,1}$ ), (center $\rightarrow$ $S_{2,1} \rightarrow S_{4,1}$ ), and (center $\rightarrow S_{2,2}$ ) for caregivers 1,2 , and 3 , respectively.

Figure 2(a) illustrates departure time variations during the simulation time, and Figure 2(b) describes corresponding arrival time variations for each task of a resident. It is shown that departure times on all tasks reach their steady states after about 600 iterations, finally deciding the crew schedule based on the chronological sequence of these departure times.

In the second data set, caregiver preference and skill set constraints result in freedom to choose corresponding caregivers without considering temporal conditions. In particular, we assume that resident 3 needs a professional skill which can be provided by caregiver 3 , and resident 5 has a personal preference for caregiver 2. Consequently, in Figure 3, it is shown that each requested caregiver is assigned to each resident accordingly at phase 2 and 6 by assignment rules 3 and 2, respectively. Other crew schedules are also decided properly, considering both spatial and temporal conditions. The final crew schedules are (center $\left.\rightarrow S_{1,1} \rightarrow S_{3,1}\right)$, (center $\rightarrow S_{2,2} \rightarrow S_{5,1}$ ), and (center $\left.\rightarrow S_{2,1}\right)$ for caregivers 1,2 , and 3, respectively.

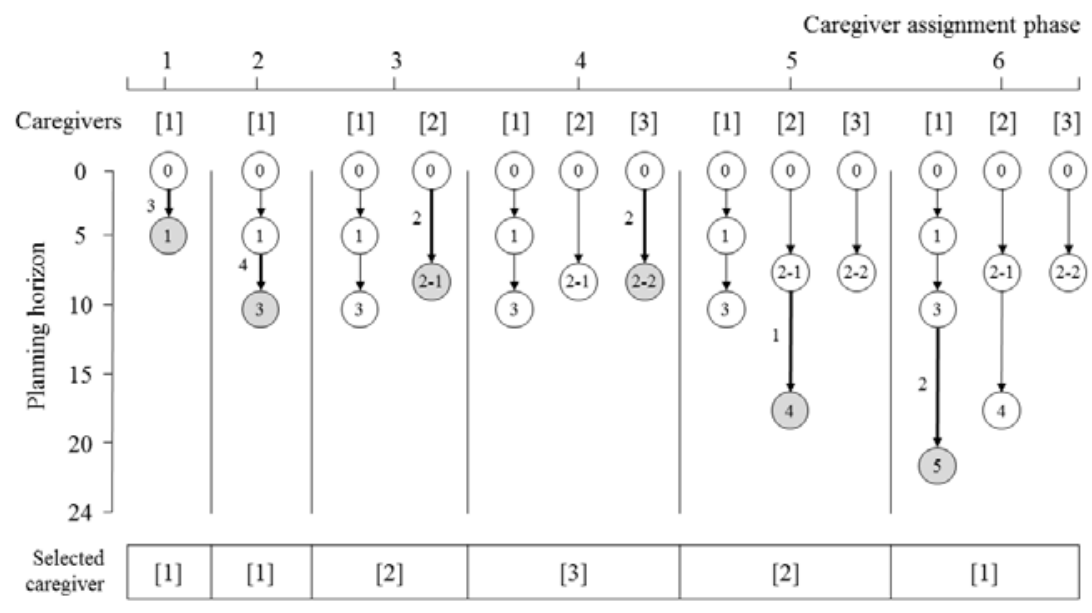

Figure 1: Simulation result (no preference and skill constraints)

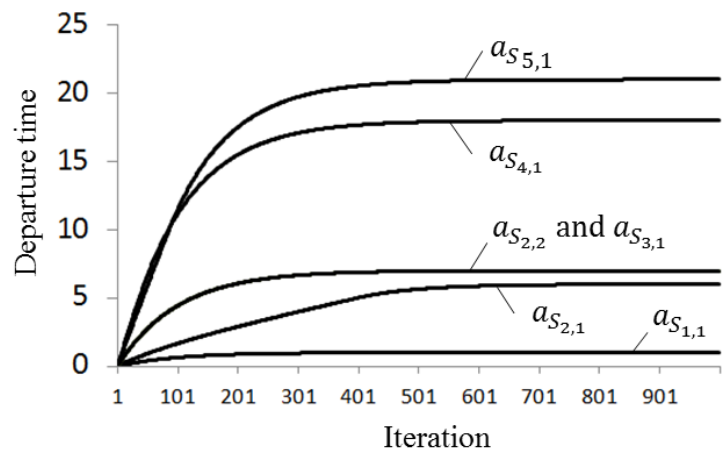

(a) Departure time variation

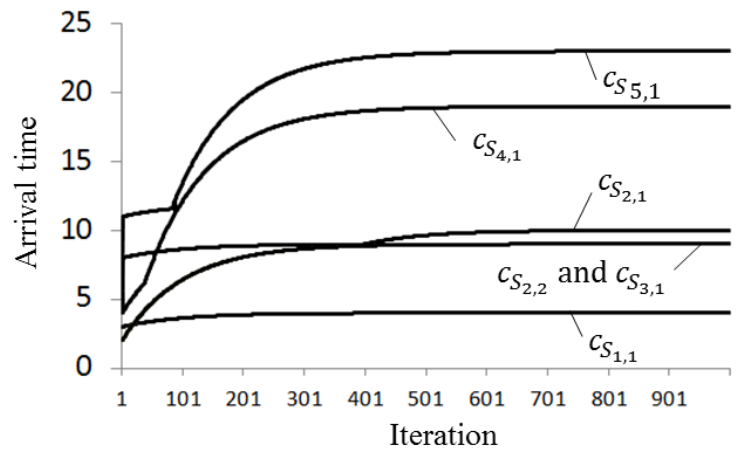

(b) Arrival time variation

Figure 2. System behaviors 


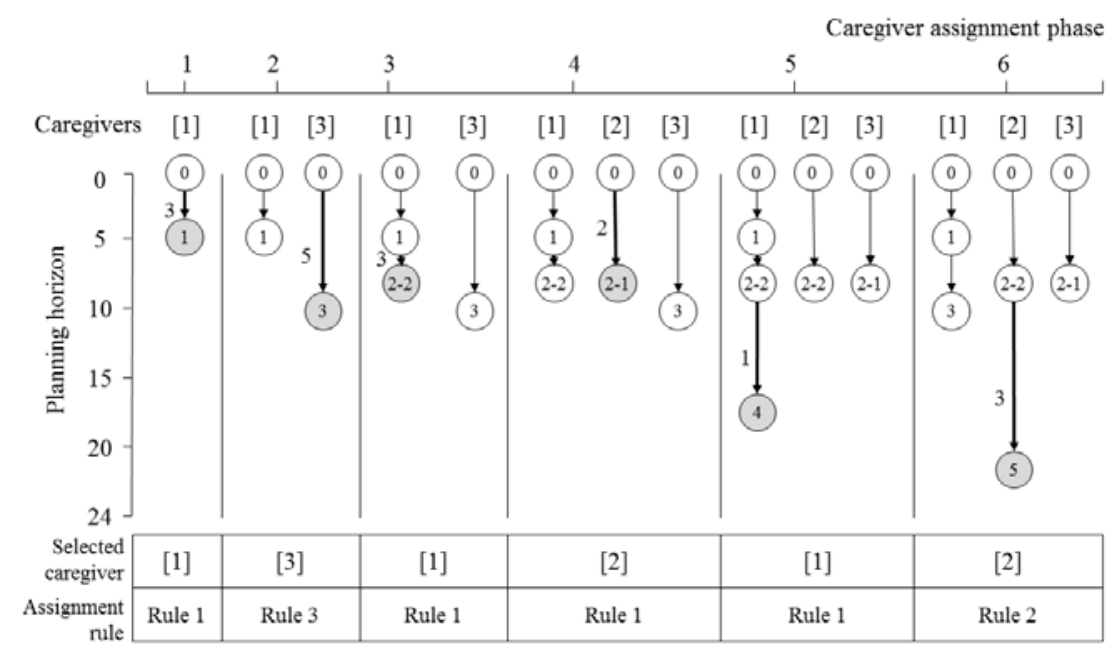

Figure 3: Simulation result (preference and skill constraints exist)

Furthermore, we test a problem set with a relatively large number of tasks of residents and evaluate the performance of the proposed feedback control algorithm, in terms of due date deviation and total travel time. We investigate 371 retirement communities located in the central Pennsylvania area, and choose 15 communities among them, distributed within about a 1-hour travel time. For each community, we randomly generate multiple (2 3) tasks so that the total number of tasks is 40 and their time windows are uniformly distributed over the 24-hour planning horizon. Total six caregivers are used, and their maximum working times are limited to about 6 hours (360 minutes) including travel times.

As a result, the routes of each caregiver are obtained as illustrated in Figure 4. The average working time of the six caregivers is about 5.72 hours (343 minutes), and their average due date deviation is 2.5 minutes. Figure 5 illustrates MSD variations through simulation, showing that it converges to its steady state (about 0.25 MSD) within an extremely short computational time ( 0.05 seconds).

\section{CONCLUSION}

In the light of the significant increase in demand for home care, HCCSP has been considered by various OR and simulation approaches across numerous studies. In this paper, we used a distributed feedback controller to schedule multiple caregivers' routings when the service starting times are given as a time window. Specifically, by building a departure controller to dynamically adjust a caregiver's departure time on task, we simulated how a routing schedule can be determined considering realistic situations between residents and caregivers. Though the results presented herein are based on a small set of data, the suggested distributed feedback controller is developed to solve complex schedule problems in a real-time manner. In this sense, the suggested controller has great potential to solve a large scale scheduling problem in a short time, compared to the other OR approaches. Therefore in future research, it will be imperative to first evaluate the efficiency of the suggested controller on a large and complex scheduling problem, and then compare with other OR approaches in terms of a "good" and "fast" scheduling to solve HCCSP. 


\section{Lee, Kang, and Prabhu}

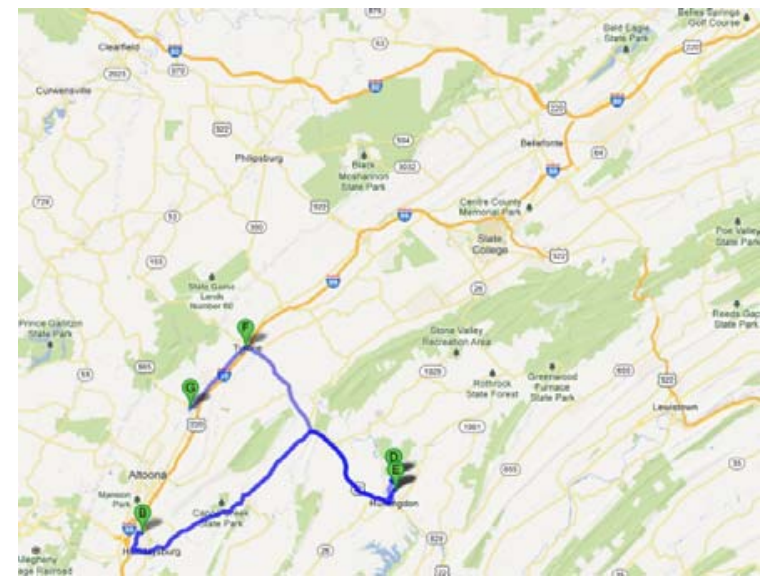

(a) Caregiver 1

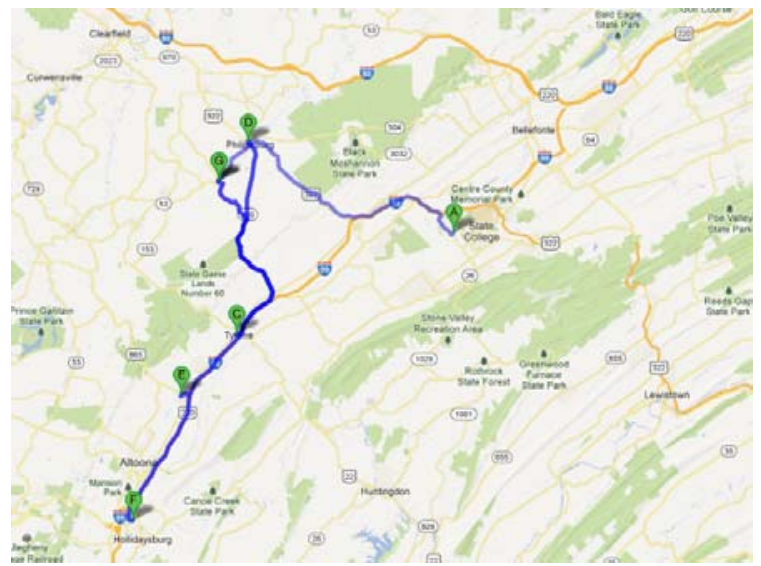

(c) Caregiver 3

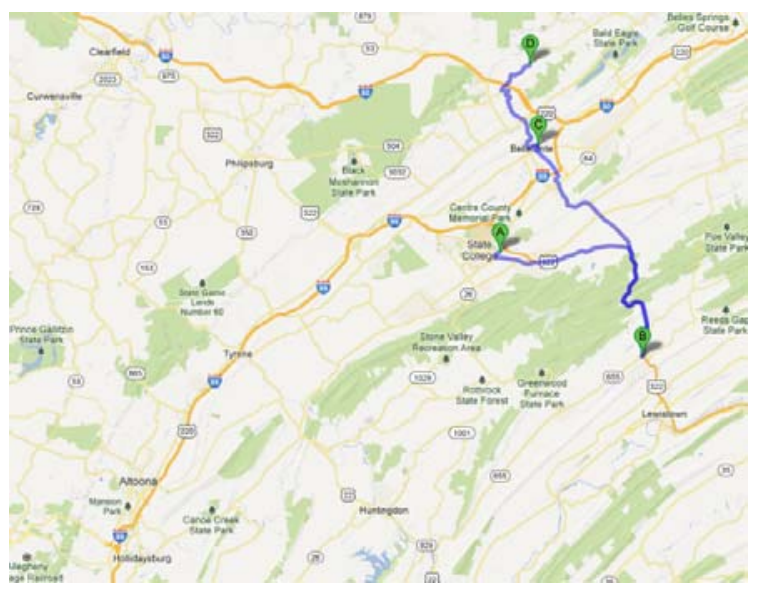

(e) Caregiver 5

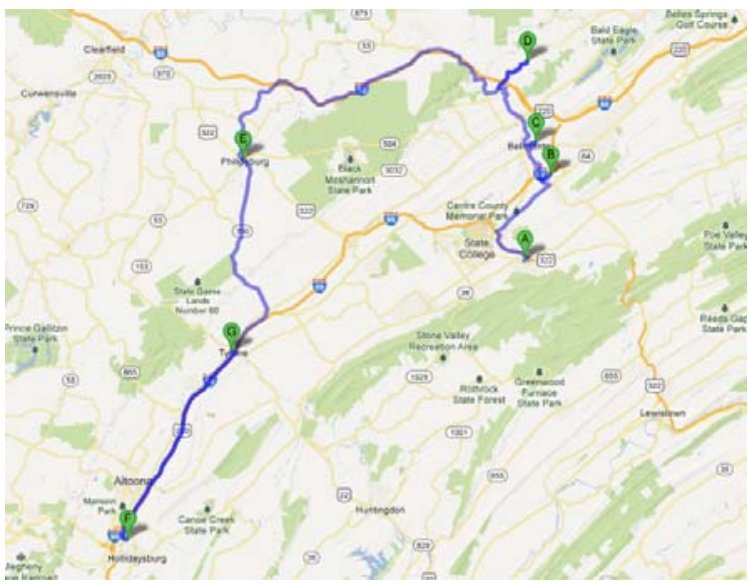

(b) Caregiver 2

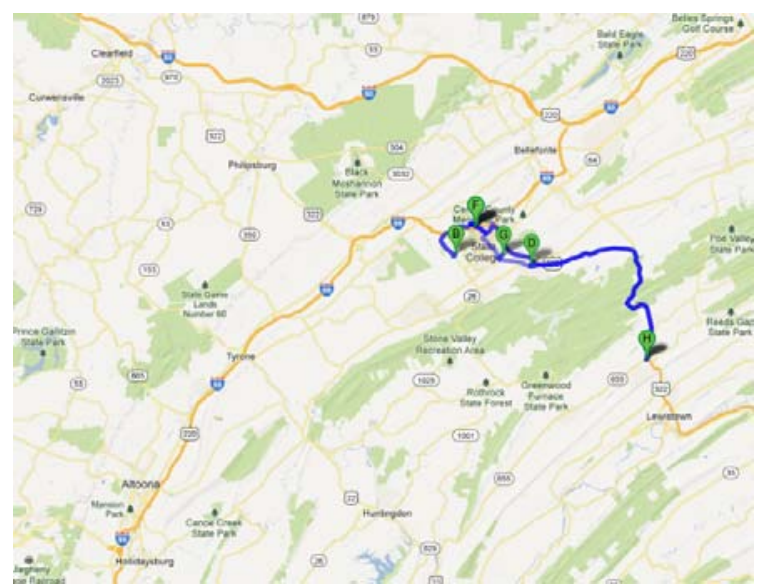

(d) Caregiver 4

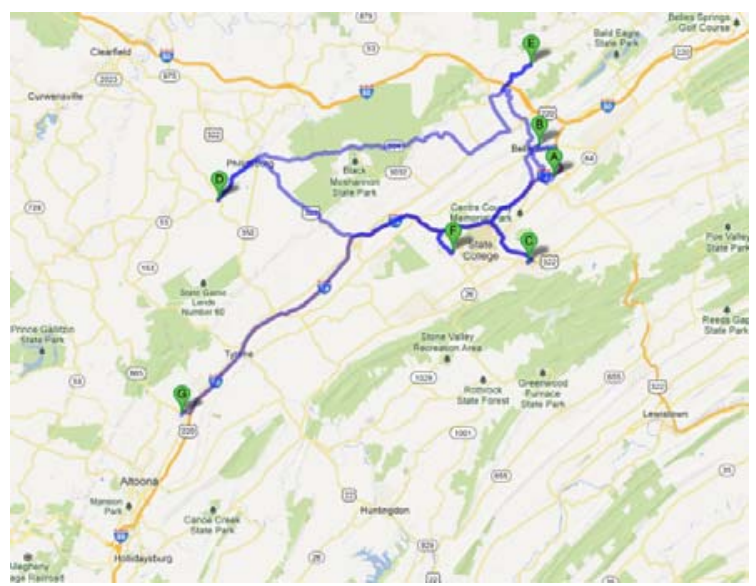

(f) Caregiver 6

Figure 4: Simulation results for 15-resident case with multiple tasks 
Lee, Kang, and Prabhu

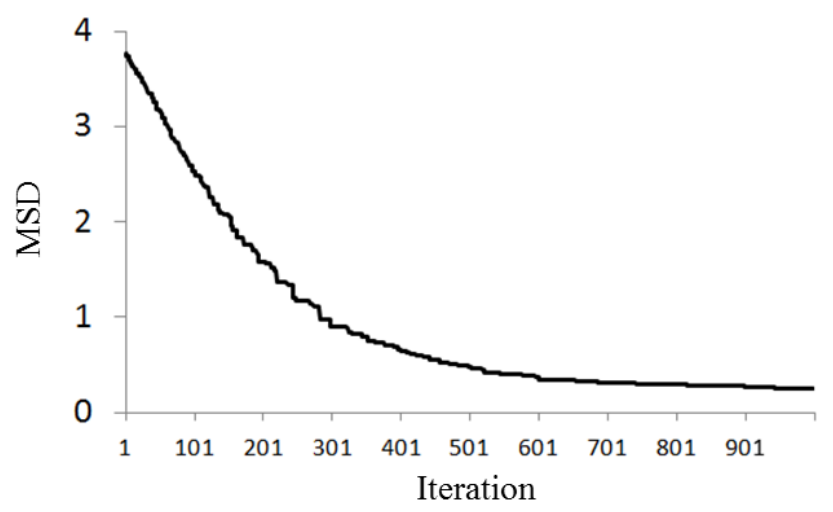

Figure 5. MSD convergence

\section{REFERENCES}

Akjiratikarl, C., P. Yenradee, and P. R. Drake. 2007. "PSO-based Algorithm for Home Care Worker Scheduling in the UK." Computers \& Industrial Engineering 53(4):559-583.

Begur, S. V., D. M. Miller, and J. R. Weaver. 1997. "An Integrated Spatial DSS for Scheduling and Routing Home-health-care Nurses." Interfaces 27(4):35-48.

Bennett, A. R. 2010. "Home health care logistics planning." Ph.D. thesis, School of Industrial and Systems Engineering, Georgia Institute of Technology, Atlanta, Georgia. https://smartech.gatech.edu/handle/1853/33989 [Accessed March 1, 2013].

Bennett, A. R. and A. L. Erera. 2011. "Dynamic Periodic Fixed Appointment Scheduling for Home Health." IIE Transactions on Healthcare Systems Engineering 1(1):6-19.

Bercovitz, A., A. J. Moss, M. Sengupta, E. Park-Lee, A. Jones, L. D. Harris-Kojetin, and M. R. Squillace. 2011 "An overview of home health aides: United States, 2007." Technical Report No. 34, US Department of Health and Human Services, Centers for Disease Control and Prevention, National Center for Health Statistics.

Bertels, S. and T. Fahle. 2006. "A Hybrid Setup for a Hybrid Scenario: Combining Heuristics for the Home Health Care Problem." Computers \& Operations Research 33(10):2866-2890.

Borsani, V., A. Matta, G. Beschi, and F. Sommaruga. 2006. "A Home Care Scheduling Model for Human Resources." In Proceedings of the 2006 International Conference on Service Systems and Service Management, 449-454.

Eveborn, P., M. Rönnqvist, H. Einarsdóttir, M. Eklund, K. Lidén, and M. Almroth. 2009. "Operations Research Improves Quality and Efficiency in Home Care." Interfaces 39(1):18-34.

Misir, M., K. Verbeeck, P. De Causmaecker, and G. V. Berghe. 2010. "Hyper-heuristics with a Dynamic Heuristic Set for the Home Care Scheduling Problem." In Proceedings of the 2010 IEEE Congress on Evolutionary Computation 1-8.

Prabhu, V. and Neil Duffie. 1995. "A Distributed Simulation Approach for Enabling Cooperation between Entities in Heterarchical Manufacturing Systems", SPIE 2596:234-242.

Rasmussen, M. S., T. Justesen, A. Dohn, and J. Larsen. 2012. "The Home Care Crew Scheduling Problem: Preference-based Visit Clustering and Temporal Dependencies." European Journal of Operational Research 219(3):598-610.

Smith, G., J. O'Keeffe, L. Carpenter, P. Doty, G. Kennedy, B. Burwell, R. Mollica, and L. Williams. 2000. Understanding Medicaid Home and Community Services: A Primer. Accessed March 1. http://www.keystonehumanservices.org/pdffiles/primerpt.htm. 


\section{AUTHOR BIOGRAPHIES}

SEOKGI LEE is currently a Ph.D. student in Industrial and Manufacturing Engineering at Penn State University, USA. His research interests include distributed control systems using feedback control theory and discrete-events simulation for service engineering processes such as supply chain management, transportation, and healthcare system management. His email address is sul201@psu.edu and his web page is https://sites.google.com/site/sgleesystem/.

YUNCHEOL KANG is currently a Ph.D. student in Industrial and Manufacturing Engineering at Penn State University, USA. He holds a master's degree in Industrial Engineering from Seoul National University. His current research interest is the use of information technology in health care and prevention education. His email address is yckang@psu.edu.

VITTALDAS V. PRABHU received his Ph.D. in Mechanical Engineering from the University of Wisconsin-Madison, USA. He is currently a Professor in Industrial and Manufacturing Engineering at Penn State University, USA. His research interests include engineering of distributed control systems consisting of discrete-events, physical processes, and service processes. He teaches courses in controls, manufacturing, information, and service systems. His email address is prabhu@engr.psu.edu and his web page is http://www.personal.psu.edu/faculty/v/x/vxp7/. 